\title{
A Scoping Review on Dental Students' Knowledge and Awareness on Radiation Protection
}

\author{
Sundaramoorthy Tamijeselvan ${ }^{1}$, Balachandra V Adkoli², Vivekanandan Ravichandran ${ }^{3}$
}

\begin{abstract}
Background: The frequent use of dental radiography is one of the major causes of the increasing medical radiation exposure of the general population. Consequently, radiation protection is a topic of scientific and public concern.

Aim: We evaluated the available research article on dental students' awareness regarding radiation protection while taking dental radiography. Materials and methods: A scoping review was performed using the available databases. A total of 452 references were found. Only five primary studies assessing the dental students' awareness about the radiation protection were included and analyzed.

Results: Knowledge of dental students about the radiation protection principle, as low as reasonably achievable (ALARA), varies from 37.4 to $70 \%$. The knowledge about the regulatory authorities like ICRP, NCRP, and AERB ranges from 5 to $54 \%$. Most of the selected articles show the knowledge about the digital radiography is below 50\% (ranges from 23 to 47\%) among the dental students. It is very surprising to know that the knowledge of the usage of protective devices like lead apron is very low among the dental students. It lies between $20 \%$ and $40 \%$. The knowledge of usage of the personnel monitoring devices among the dental students is average. It ranges between $40 \%$ and $70 \%$.

Conclusion: Knowledge gaps concerning the awareness of radiation protection among the dental students are evident from published research. The knowledge on radiation protection can be improved by conducting seminars and workshops on radiation protection on dental radiography. Keywords: Awareness, Dental students, India, Knowledge, Radiation protection.

Annals of SBV (2019): 10.5005/jp-journals-10085-8115
\end{abstract}

\section{INTRODUCTION}

Energy in a moving form is called radiation. Radiation can be classified into nonionizing radiation and ionizing radiation depending on the properties of radiation. X-radiation is one of the ionizing radiations of electromagnetic nature. X-rays play a vital role in medical diagnostics. Radiographs have become an important tool in dental diagnosis and treatment planning.

As in the case of all modern modalities, X-ray is also having both benefits and risk. The first and foremost risk of X-radiation is the biological effect. The biological effect means the effect caused by the ionizing radiation to a living organism, particularly to human being.

Radiation protection is not to completely blocking radiation. It is the way to optimize the use with minimal exposure. As low as reasonably achievable (ALARA) is the basic principle of radiation protection. As a dental student, the awareness of radiation protection is essential to protect the individual and the patient. In this review, we are going to study the awareness of radiation protection among dental students in India.

\section{BACKGROUND}

Radiography is a diagnostic test, and in dentistry, this test almost always involves the use of ionizing radiation. It includes intraoral periapical (IOPA) radiograph, orthopantomography (OPG), and cone-beam computed tomography (CBCT). It is essential that oral and maxillofacial radiography should be performed thoughtfully and responsibly, with minimizing radiation dose but maximizing diagnostic benefit. ${ }^{1}$ As low as reasonably achievable is not only a safety principle but also a regulatory requirement for all radiation safety programs. ${ }^{2}$
${ }^{1}$ Department of Radiography, Mother Theresa Post Graduate and Research Institute of Health Sciences, Puducherry, India

${ }^{2}$ Department of Medical Education, Sri Balaji Vidyapeeth (Deemed to be University), Puducherry, India

${ }^{3}$ Department of Radiology, Indira Gandhi Government General Hospital and Postgraduate Institute, Puduchery, India

Corresponding Author: Sundaramoorthy Tamijeselvan, Department of Radiography, Mother Theresa Post Graduate and Research Institute of Health Sciences, Puducherry, India, Phone: +91 9443208730, e-mail: tamije1970@gmail.com

How to cite this article: Tamijeselvan S, Adkoli BV, Ravichandran V. A Scoping Review on Dental Students' Knowledge and Awareness on Radiation Protection. Ann SBV 2019;8(2):26-29.

Source of support: Nil

Conflict of interest: None

There are many regulatory authorities at national and international levels to regulate the medical use of ionizing radiation. The International Commission on Radiological Protection (ICRP), National Council on Radiation Protection Measurements (NCRP), and Atomic Energy Regulatory Board (AERB) have established guidelines regarding the specifications of $X$-ray equipment, room layout of X-ray installation, protective devices, and personnel safety. ${ }^{3,4}$

The regulations are there for the radiation protection. But the awareness has to be improved among the operators and the physicians who are referring the investigation based on the ionizing radiation. There is definite scarcity of literature exploring knowledge of radiation protection among dental professionals,

() The Author(s). 2019 Open Access This article is distributed under the terms of the Creative Commons Attribution 4.0 International License (https://creativecommons. org/licenses/by-nc/4.0/), which permits unrestricted use, distribution, and non-commercial reproduction in any medium, provided you give appropriate credit to the original author(s) and the source, provide a link to the Creative Commons license, and indicate if changes were made. The Creative Commons Public Domain Dedication waiver (http://creativecommons.org/publicdomain/zero/1.0/) applies to the data made available in this article, unless otherwise stated. 
students, and radiographers in the Indian context. ${ }^{5}$ The use of ionizing radiation can be regularized by following the radiation protection principle. The main component of the principle is justification, optimization, and dose limit. The radiation exposure amount from dental radiographs depends on variable factors like speed of film, technical factors, the technique used to take radiograph, type of collimators, and the use of protective barrier such as lead apron. ${ }^{6}$

The quality and quantity of the X-ray used for taking radiography depends on the tube potential $(\mathrm{kVp})$, the tube current $(\mathrm{mA})$, and time of exposure (s). The lack of awareness about tube potential $(\mathrm{kVp})$ may be attributed to the fact that conventional dental X-ray machines in India are having fixed $k V p$ and tube current $(\mathrm{mA})$ operating at $70 \mathrm{kVp}$, and the only variable factor in the dental unit is the exposure timer (s). ${ }^{7}$ It is generally considered that risk from the diagnostic procedure using $X$-rays are small when compared to the radiation therapy. Also the health risks to individuals are also small. But, the growing number of people exposed to X-ray makes low level of X-radiation also a major cause of concern when it is calculated as a cumulative dose. ${ }^{8}$

The shape, size, and intensity of the X-ray beam have been reported as the most important factors in determining the radiation dose to the patient in the dental radiography. For intraoral periapical radiography, limitation of the beam was more with rectangular collimator compared to conical. ${ }^{9}$ So, it is advised to use a rectangular collimator for taking IOPA views. Currently, modern technologies have been introduced such as the utilization of digital panels, which is rapidly enhancing and now coming to the market. Some of the main benefits of using digital radiography are that it can be used for image enhancement and referral purposes. The main advantage and most paramount is that it may reduce the radiation exposure. $^{10,11}$

The devices used for the measurement of radiation dose is called dosimeter. Personnel monitoring dosimeters such as film badge and thermoluminescent dosimeter (TLD) are used to measure the radiation received by a radiation worker. The dose from the various types of radiation is measured by these devices and are processed and reported to the employer by the dosimetry service provider. ${ }^{12}$ The knowledge about the radiation protection in dental radiography is very essential for dental students to limit the dose for themselves as well as to the patients.

\section{A IM}

The aim of this scoping review was to search for all available articles on assessing dental students' awareness on radiation protection and radiation risks of dental radiography. The review should answer the following questions: (1) do the dental student knows about the radiation doses of dental radiography and the radiation protection principle (ALARA)? (2) Are dental students aware of the rules and regulations framed by the regulatory authorities such as ICRP, NCRP, and AERB? (3) Are the dental student aware of the possible biological effect of radiation particularly to the pregnant women? (4) Do the dental students know the benefit of using digital radiography and collimators? (5) Do the dental students know the usage of the personnel radiation protection devices such as lead apron?

\section{Materials and Methods}

Between January 2017 and January 2020, we comprehensively searched Google Scholar, WebMD, and ProQuest. We used combinations of medical subject headings and free text words that included search terms related to the exposure such as knowledge, radiation hazards, protection, dental students, and India, which were combined with search terms related to the outcomes. We identified articles eligible for further review by performing an initial screen of identified titles or abstracts, followed by a full-text review (Flowchart 1).

\section{Results and Discussion}

Five important concepts are identified for the review. They are (1) knowledge about radiation protection principle-ALARA; (2) knowledge about the regulatory authorities like ICRP, NCRP, and AERB; (3) knowledge about the digital radiography, collimator, and speed of the film; (4) knowledge about the protective barriers such as lead apron; and (5) knowledge about the usage of personnel monitoring devices such as film badge and thermoluminescent dosimeter (TLD) (Table 1).

\section{Knowledge about Radiation Protection Principle-ALARA}

The important principle for radiation protection is ALARA. This principle defines us how to take a reasonably good radiographic image with minimum possible radiation. The data from the selected five articles are analyzed with respect to the knowledge of dental students regarding the radiation protection principle. The percentage of the knowledge varies from 37.4 to $70 \%$.

\section{Knowledge about the Regulatory Authorities Like ICRP, NCRP, and AERB}

There are several regulatory authorities for regulating the rules for radiation protection. At international level, International Commission for Radiological Protection (ICRP), and at national level, National Commission for Radiological Protection (NCRP) and Atomic Energy Regulatory Board are doing the regulation for radiation protection. The selected five articles were analyzed with respect to the above concept and found that the knowledge of dental students regarding the regulatory authorities such as ICRP, NCRP, and AERB ranges from 5 to $54 \%$.

Flowchart 1: Process of selection of the article for this review

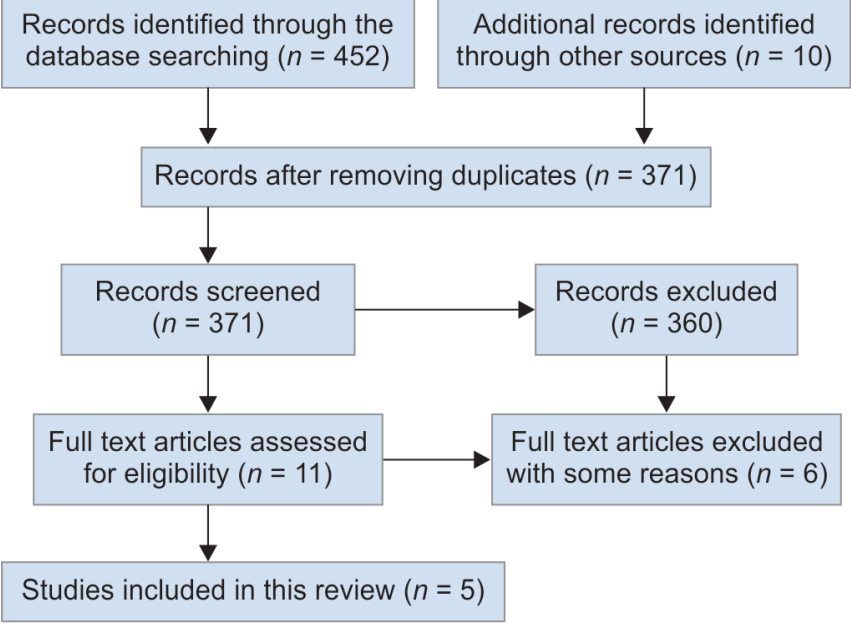


Table 1: Studies included in this review

\begin{tabular}{lll}
\hline Author, country, journal & Aim & Methodology \\
\hline Motwani Mukta, India, & To assess the knowledge & A validated 20-point \\
International Journal of & and attitudes regarding \\
radiation hazards and & questionnaire about radiation \\
protection among medical & protocol in the form of multiple \\
& and dental students & $\begin{array}{l}\text { choices was used for the study to } \\
\text { collect data. }\end{array}$
\end{tabular}

Rahul Srivastava, India, To evaluate the knowledge, Journal of International Oral attitude, and perception Health

(KAP) of dental students toward ionizing radiation, biological hazards, and appropriate radiographic protection methods

Rathi Rela, India, Dentistry

To assess the knowledge, attitude, and practice of radiation protection among dental students in a dental college in eastern India

K Roja Reddy, India, Acta Scientific Dental Sciences

Madhu Sudhan Vasantharajan, India, Biomedical \& Pharmacology Journal

To evaluate the knowledge and awareness toward radiation hazards and radiation

To assess the knowledge, attitude, and practice of undergraduate dental students on radiation
A cross-sectional questionnaire study was conducted. A total of 174 dental students who had completed their 30 days' posting in Department of Oral Medicine and Radiology were inducted for the study. All the students were subjected to the structured 17-response questionnaire with multiple choices. Data collected were subjected to statistical analysis using the SPSS software package version 17 .

A cross-sectional survey was conducted among 107 dental students comprising of undergraduate students, dental interns, and postgraduate students in a teaching dental institute in capital city in eastern Indian state. exposure protection for pedodontic patients

A questionnaire comprising 25 structured questions were distributed and data were collected.

\section{A self-administered} questionnaire consisting of 13 questions with both "yes," "no," and multiple-choice pattern was prepared to obtain information about knowledge, attitude, and
Summary of findings

There is a need "to fill" the knowledge deficit for students from dental fraternity, thereby creating awareness about radiation hazards and protection. There is a need to educate future dentists regarding unnecessary exposure of individual to radiation. ${ }^{13}$

The results from the present study revealed that the KAP level in regard to the biological hazardous effects of $X$-ray and protection was low to average among dental students. It is recommended that the syllabus of dental radiology should be expanded to provide clinical knowledge regarding radiation hazards and protection so that students are well grounded with the principles of dental radiography. ${ }^{14}$ practice on radiation exposure protection for pedodontic patients.
Based on results of this finding, the overall knowledge, awareness, and practice regarding radiation protection among dental student were not uniformly good. ${ }^{15}$

The results of the study revealed that the knowledge and awareness regarding radiation safety and protection were less among the dental students. ${ }^{16}$

To conclude, it can be noted that the final-year dental students were much more knowledgeable in using radiograph judicially compared to the other groups of participants, which belong to thirdyear dental students. However, in terms of practice, both third-year and final-year dental students irrespective of year of study do not follow radiation protection measures, even though they were aware of it. ${ }^{17}$

\section{Knowledge about the Digital Radiography, Collimator, and Speed of the Film}

Optimization is a technique that maximizes the use of radiation for taking a radiograph. This plays a vital role in the radiation protection. Digital radiography, collimation, and speed of the film are the major tools for optimization. The knowledge about using the technique is very essential for radiation protection. Most of the selected articles show the knowledge about the digital radiography is below $50 \%$ (ranges from 23 to $47 \%$ ) among the dental students.

\section{Knowledge about the Protective Barriers such as Lead Apron}

Shielding plays a vital role in the radiation protection. Shielding with the lead apron protects one from the unnecessary radiation. The operator should use this radiation protection barriers to protect themselves. It is very surprising to know that the knowledge of the usage of protective devices like lead apron is very low among the dental students. It lies between 20 and $40 \%$

\section{Knowledge about the Usage of Personnel Monitoring Devices such as Film Badge and TLD}

The personnel monitoring device is used to monitor the radiation received by an individual during his lifetime. It is simply cumulative dose received by an individual. There are two personnel monitoring devices currently in use, namely film badge and TLD. The knowledge of usage of the personnel monitoring devices among the dental student is a must. On reviewing the article, we found that the knowledge about the usage of personnel monitoring devices is found to be average. It ranges between 40 and $70 \%$.

\section{Conclusion}

The review shows that the overall knowledge level among the dental students in India with respect to the radiation protection 
was low to average. This shows that there is a necessity for more theoretical and practical training of the undergraduate dental students for radiation safety protocols. This will lead to increase in knowledge about the radiation protection among the dental radiography. This can also be achieved by conducting seminars and workshops on radiation protection.

\section{References}

1. Lam EW. Considerations for the Use of lonizing Radiation in Dentistry. Royal College of Dental Surgeons of Ontario; 2011.

2. Radiation Safety and ALARA. Environmental Health \& Safety Center Radiation Safety Division 515-2894. NC State University.

3. Hagi SK, Khafaji MA. Medical students' knowledge of ionizing radiation and radiation protection. Saudi Med J 2011;32(5):520-524.

4. Grover SB, Kumar J, Gupta A, Khanna L. Protection against radiation hazards: regulatory bodies, safety norms, does limits and protection devices. Indian J Radiol Imaging 2002;12(1):157-167.

5. Binnal A, Rajesh G, Denny C, Ahmed J, Nayak V. Insights into the state of radiation protection among a subpopulation of Indian dental practitioners. Imaging Sci Dent 2013;43(4):253-259. DOI: 10.5624/ isd.2013.43.4.253.

6. Asha, Veena SN, Krupashankar R, Kavitha AP, Shobha R, Jijin MJ, et al. Awareness towards radiation protection measures among dental practitioners in Coorg district: a questionnaire study. Int J Dent Health Sci 2015;2(6):1460-1465.

7. Agrawal B, Dosi T, Hazari A, Maheshwari C, Rajput Y, Nidhi. Evaluation of radiation protection awareness amongst general dental practitioners of western Rajasthan in India. J Int Oral Health 2015;7(12):S1-S5.

8. Yurt A, Cavusoglu B, Gunay T. Evaluation of awareness on radiation protection and knowledge about radiological examinations in healthcare professionals who use ionized radiation at work. Mol Imaging Radionucl Ther 2014;22(2):48-53. DOI: 10.4274/mirt. 00719.

9. Swarna YM, Devarasa GM, Annigeri R, Kalra D. Compliance of Indian dentists with oral radiology safety measures. J Oral Maxillofac Radiol 2013;1(3):104-110. DOI: 10.4103/2321-3841.126744.

10. Wenzel A, Gröndahl H. Direct digital radiography in the dental office. Int Dent J 1995;45(1):27-34.

11. Parks ET, Williamson GF. Digital radiography: an overview. J Contemp Dent Pract 2002;3(4):23-39. DOI: 10.5005/jcdp-3-4-23.

12. Personal Radiation Monitoring - Information for Employers and Employees; 2013.

13. Mukta M, Pooja T, Apeksha D, Apurva K. Knowledge and attitude amongst the dental and medical students towards radiation hazards and radiation protection: a questionnaire survey. Int J Dent Res 2019;4(2):43-48.

14. Srivastava R, Jyoti B, Jha P, Shukla A. Knowledge, attitude, perception toward radiation hazards and protection among dental undergraduate students: a study. J Int Oral Health 2017;9(2):81-87. DOI: 10.4103/jioh.jioh_26_16.

15. Rela R. Knowledge, attitude and practice of radiation protection protocols amongst students of a dental college. Dentistry 2019;9(1):530. DOI: 10.4172/2161-1122.1000530.

16. Reddy R, Krishnan M, Ramesh T, Krishna S, Swathi G, Praveen KNS. Evaluation of knowledge and awareness on practice of dental radiographic safety measures in West Godavari District, India-a questionnaire based cross sectional study. Acta Sci Dent Sci 2017;1(2):3-9.

17. Rahman FBA, Gurunathan D, Vasantharajan MS. Knowledge, attitude and practice of radiation exposure protection for pediatric patients among undergraduate dental students. Biomed Pharmacol J 2018;11(2):1143-1151. DOI: 10.13005/bpj/1475. 